\title{
Litofacies y mineralogía de los depósitos de sínter de la zona geotérmica de San Agustín del Maíz, Michoacán, México
}

\author{
Emmanuel Olvera-García ${ }^{1,2, *}$, Víctor Hugo Garduño-Monroy ${ }^{1}$, \\ Mikhail Ostrooumov ${ }^{1}$, Gerardo Bermejo-Santoyo ${ }^{1}$, \\ Jorge Alejandro Guevara-Alday ${ }^{3}$, Andrea Brogi' ${ }^{2}$ y \\ Domenico Liotta ${ }^{2}$
}

${ }^{1}$ Universidad Michoacana de San Nicolás de Hidalgo, Instituto de Investigación en Ciencias de la Tierra, Morelia, Michoacán, México.

${ }^{2}$ Università degli Studi di Bari, Dipartimento di Scienze della Terra e Geoambientali, Bari, Italia.

${ }^{3}$ University of Quebec in Montreal, Department of Earth and Atmospheric Science, Montreal, Canada.

*emmanuelog_08@outlook.com

\section{RESUMEN}

En la zona geotérmica de San Agustín del Maíz (Michoacán) se han identificado depósitos de sínter de sílice coexistiendo con manifestaciones hidrotermales activas. Estos depósitos, considerando el modo de deposición, se separan en bandeados de fractura y estratificados. Los primeros están asociados a los conductos a través de los cuales los fluidos ascienden a la superficie, mientras que los segundos se forman a partir de los fluidos que logran llegar a la superficie. Los depósitos estratificados presentan diferentes litofacies que se pueden agrupar en un rango con dos miembros finales: por un lado, un depósito hidrotermal puro, denso, de aspecto vítreo y compacto. Por el otro lado, un depósito hidrotermal limo-arenoso, deleznable, con impresiones de plantas y algunos líticos. El depósito vítreo se considera geiserita, formado por salmueras con temperaturas superiores a $\operatorname{los} 70^{\circ} \mathrm{C}$, mientras que el depósito limo-arenoso se considera que se ha formado por salmueras con temperaturas inferiores a $\operatorname{los} 70^{\circ} \mathrm{C}$ y en algunos casos, distantes de la surgente termal. Los depósitos vítreos están constituidos mineralógicamente solo por ópalo (C y CT), mientras que los depósitos limo-arenosos están formados por ópalo en conjunto con otras fases mineralógicas asociadas a sedimentos. Se ha establecido que las litofacies y la mineralogía de los depósitos de sínter de sílice pueden dar información de las temperaturas y las tasas de deposición de las surgentes hidrotermales que les dieron origen. Esta información es de utilidad para la identificación de áreas donde existió mayor emergencia de fluidos hidrotermales a la superficie y con mayor temperatura (e.g. facies de geiserita), especialmente en zonas geotérmicas con un contexto geológico similar al de San Agustín del Maíz (e.g. sector central del Cinturón Volcánico Trans-Mexicano). Dichas áreas con importante circulación de fluidos pueden coincidir localmente con intersección de fallas, terminación de fallas y rampas de relevo.

Palabras clave: sínter de sílice; ópalo; litofacies; mineralogía.

\begin{abstract}
In the active San Agustín del Maíz geothermal zone (Michoacán), we identified silica-sinter deposits co-existing with hot springs. Considering the deposition mode, these deposits are separated into fracture-banded and bedded deposits. The first ones are associated with deposition on the channels from the fluids rising to the surface, while the second ones are formed from the geothermal fluids that reached the surface. The bedded deposits showed different types of lithofacies grouped in a range with two endmembers: on the one hand, a pure silica-sinter deposit characterized by high density, glassy aspect and being consolidated. On the other hand, a silty-sandy deposit, that easily disaggregate, presenting impression of plants and lithics. The vitreous deposit is considered geyserite, formed from fluids with temperatures superior to $70^{\circ} \mathrm{C}$, while the silty-sandy deposits are formed from fluids with temperatures below $70{ }^{\circ} \mathrm{C}$ and, in some cases, far away from the water source. The geyserite deposits are mineralogically composed only by opal (C and CT), while the silty-sandy deposits are formed by opal in association with other mineralogical phases (linked to sediments). Consequently, we recognized that the lithofacies and the mineralogy of the silica-sinter deposits give information of the temperatures and deposition rates of the parental fluids. This information could be very useful in order to identify setting where existed grater geothermal up flow and temperature (e.g. geiserite
\end{abstract}


facies) in areas with similar geological context to San Agustín del Maíz (e.g. central sector of the Cinturón Volcánico Trans-Mexicano). These sceneries can be associated to structural settings like fault intersections, fault-tips and relay-ramps.

Key words: silica-sinter; opal; lithofacies; mineralogy.

\section{INTRODUCCIÓN}

En las zonas geotérmicas activas de alta temperatura, las manifestaciones hidrotermales en superficie pueden tomar forma de manantiales termales, manantiales hirvientes, fumarolas, géiseres, cráteres de explosión freática, zonas de alteración (Wohletz y Heiken, 1992) y volcanes de lodo. Además, es común que a partir de los fluidos geotérmicos que llegan a la superficie mediante la deposición química se formen depósitos hidrotermales de sílice y/o calcita conocidos como sínter (e.g. Herdianita et al., 2000; Pentecost, 2005; Brogi et al., 2012; Guidoy Campbell, 2011; Canet et al., 2019). Estos depósitos de sínter sirven como modelos para el entendimiento de procesos en la parte más somera de un sistema hidrotermal (Walter et al., 1996; Herdianita et al., 2000; Renaut y Jones, 2003; Guido y Campbell, 2011) y pueden ser importantes durante la exploración geotérmica y minera (Cunneen y Sillitoe, 1989; Herdianita et al., 2000; Lynne, 2012; Renaut y Jones, 2003; Campbell et al., 2015).

Dentro de los depósitos de sínter, los depósitos de sílice son los más comunes, formándose en zonas continentales y volcánicamente activas (Campbell et al., 2015), donde la interacción entre fluidos meteóricos y cuerpos magmáticos en enfriamiento (incluyendo fluidos magmáticos) da lugar a la formación de fluidos geotérmicos. Estos fluidos, bajo altas presiones y temperaturas en el subsuelo, disuelven la sílice de las rocas encajonantes (Pentecost, 2005). Estos fluidos ricos en sílice llegan a la superficie comúnmente a lo largo de fallas (Curewitz y Karson,
1997; Rowland y Sibson, 2004; Campbell et al., 2015; Olvera-García et al., 2020a, 2020b) y depositan la sílice alrededor de las surgentes termales debido al enfriamiento, la evaporación y los cambios de $\mathrm{pH}$ (Boudreau y Lynne, 2012). En la superficie los fluidos que forman el sínter se caracterizan por ser casi neutros, de tipo cloruradas-sódicos y con temperaturas inferiores a los $100{ }^{\circ} \mathrm{C}$ (Fournier y Rowe, 1966; Herdianita et al., 2000; Campbell et al., 2015).

En la zona geotérmica de San Agustín del Maíz (SAM) se identificaron depósitos de sínter de sílice coexistiendo con manifestaciones hidrotermales activas que alcanzan temperaturas de hasta $93^{\circ} \mathrm{C}$. Los depósitos hidrotermales identificados se separan en depósitos bandeados y depósitos estratificados. Estos últimos presentan diferentes litofacies y mineralogía. La mineralogía de estos depósitos está representada principalmente por ópalo $\left(\mathrm{SiO}_{2} \mathrm{n} \mathrm{H}_{2} \mathrm{O}\right)$ en muchos casos coexistiendo con otras fases mineralógicas. En este trabajo se asocian las litofacies y la mineralogía de los depósitos hidrotermales identificados, con la intención de relacionarlos con la posible temperatura de los fluidos que les dieron origen y con los escenarios geológicos favorables para la circulación de los fluidos hidrotermales. El principal objetivo es usar los depósitos hidrotermales de alta temperatura como una herramienta para identificar escenarios donde existió mayor circulación de fluidos a la superficie, con mayores temperaturas, en regiones con contextos geológicos-estructurales similares al de SAM.

\section{CONTEXTO GEOLÓGICO}

La zona de estudio se encuentra localizada en el sector central de la provincia geológica conocida como Cinturón Volcánico TransMexicano (CVTM) (Figura 1a), la cual es un arco volcánico del Neógeno-Cuaternario (Demant, 1978; Pasquaré et al., 1991; GómezTuena et al., 2007, Ferrari et al., 2012). Este escenario geológico dio lugar a una sucesión volcano-estratigráfica representada por; 1) una

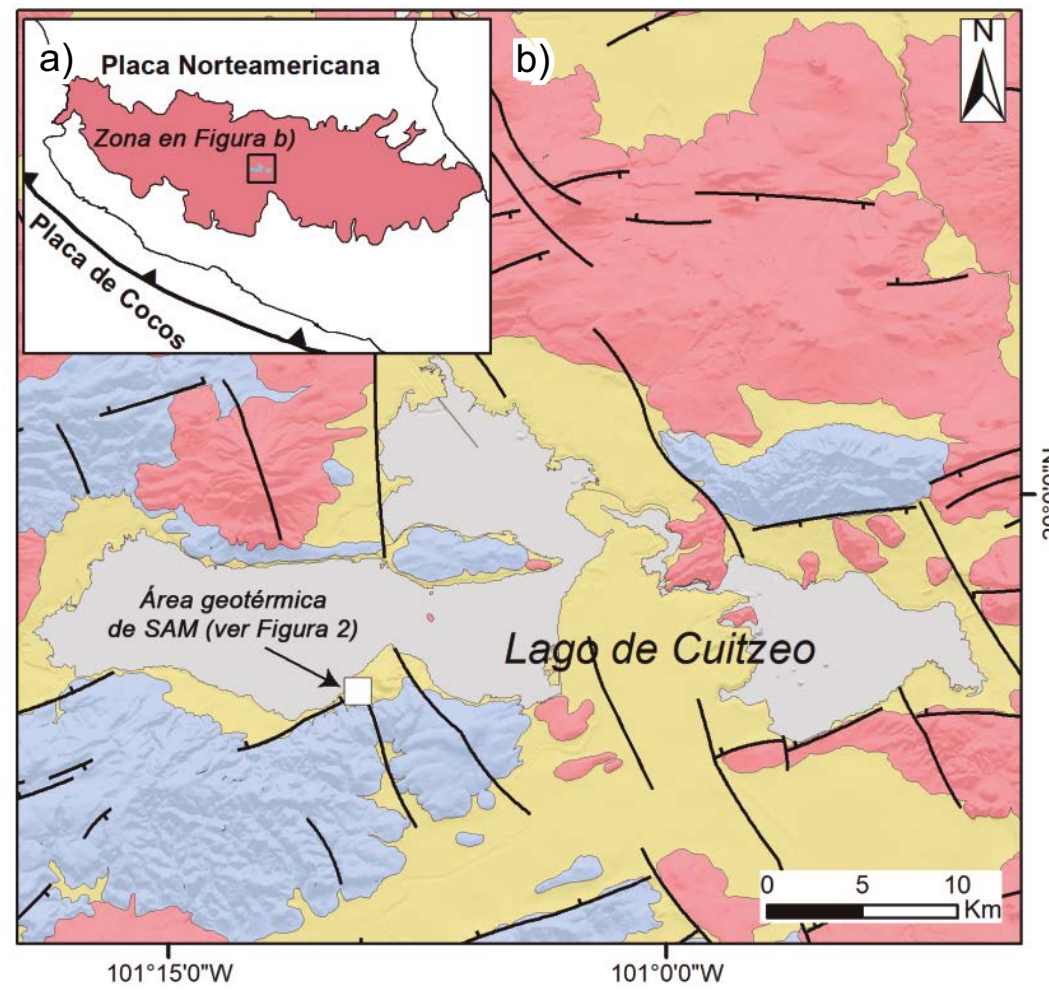

3. Depósitos lacustres y aluviales Mio-Holocénicos

2. Volcanes y productos volcánicos (de ácidos a básicos) del Mioceno tardío al Holoceno

1. Lavas (andesíticas y basálticas) y depósitos ignimbríticos del Mioceno medio y tardío

Fallas de normal a oblicua izquierda Falla de lateral a oblicua

Figura 1. Contexto tectónico y geológico del área de estudio; a) zona de estudio en el marco del Cinturón Volcánico TransMexicano (polígono rosa); b) contexto geológico del área de estudio. 
secuencia del Mioceno medio y tardío, formada por lavas andesíticas y basálticas, así como depósitos ignimbríticos (Figura 1b), cubierta por 2) una secuencia del Mioceno tardío al Cuaternario conformada por volcanes semi-escudo, domos riolíticos y volcanes monogenéticos (e.g. Gómez-Tuena et al., 2007; Gómez-Vasconcelos et al., 2015), estos últimos asociados al Campo Volcánico Michoacán-Guanajuato (e.g. Johnson et al., 2009). Finalmente, desde el Mioceno tardío las secuencias previas se han venido intercalando con 3) depósitos lacustres y aluviales (Figura 1b) (Israde-Alcántara, 1997; Israde-Alcántara y Garduño-Monroy, 1999).

La zona geotérmica de San Agustín del Maíz (SAM) en Michoacán, se ubica en la ribera sur de la cuenca tectónica del Lago de Cuitzeo (Figura 1), localizada a $30 \mathrm{~km}$ al norte de Morelia (Michoacán). Esta zona es afectada por una tectónica regional con una extensión de dirección aproximada NO-SE (Pasquaré et al., 1988; Suter et al., 1991, 2001; Ego y Ansan, 2002), la cual ha permitido la formación de fallas normales de dirección SO-NE y OSO-ENE, así como fallas laterales de dirección NNO-SSE; estas últimas juegan el papel de fallas de transferencia (Figura 1b) (Olvera-García et al., 2020a). Este escenario ha permitido la formación de cuencas tectónicas orientadas SW-NE, donde se han depositado los sedimentos lacustres antes mencionados (e.g. Israde-Alcántara, 1997; Israde-Alcántara y Garduño-Monroy, 1999).

\section{METODOLOGÍA}

Se llevó a cabo un mapeo de la distribución de los depósitos hidrotermales que se encuentran en la comunidad de SAM (Michoacán), describiendo también su morfología. Posteriormente se realizó la descripción de las texturas y de las litofacies de estos depósitos. De la misma forma, se efectuó un muestreo de cuatro depósitos hidrotermales con diferentes litofacies para la realización de análisis mineralógicos.

Las muestras de los depósitos hidrotermales fueron trituradas hasta obtener un tamaño de $40 \mu \mathrm{m}$. Posteriormente, con el fin de caracterizarlos, se ejecutaron estudios mineralógicos de las muestras en polvo con el método de difracción de rayos X (DRX) y espectroscopía infrarroja en los laboratorios del Instituto de Ciencias de la Tierra (INICIT) de la Universidad Michoacana de San Nicolas de Hidalgo (UMSNH).

Los diagramas de DRX de las muestras analizadas fueron generados usando un difractómetro Siemens XRD 5000. Las condiciones de operación fueron radiación $\mathrm{Cu} K \alpha(\lambda=1.5406 \AA), 40 \mathrm{kV}, 30 \mathrm{~mA}$, con resolución de $0.01^{\circ}$, y un intervalo de registro de $2 \Theta=5.0-70.0^{\circ}$. Los diagramas fueron registrados en un modo de escaneo gradual con pasos de $0.02^{\circ} 2 \Theta$ y una tasa de conteo de 100 espectros por paso. Los análisis mineralógicos cuantitativos fueron llevados a cabo mediante el software DIFFRAC PLUS (compañía BRUKER AXS).

\section{RESULTADOS}

En la zona geotérmica de SAM se encontraron 182 manifestaciones hidrotermales superficiales con un intervalo de temperatura que va de 32 a $93{ }^{\circ} \mathrm{C}$ (Figura 2). Estas manifestaciones están representadas principalmente por manantiales termales (Figura 3a-3c), encontrando también algunos puntos con emisiones de vapor y emisiones de gas, que en algunos casos, dan lugar a la formación de pequeños volcanes de lodo. Además, asociados a los manantiales termales, se identificaron

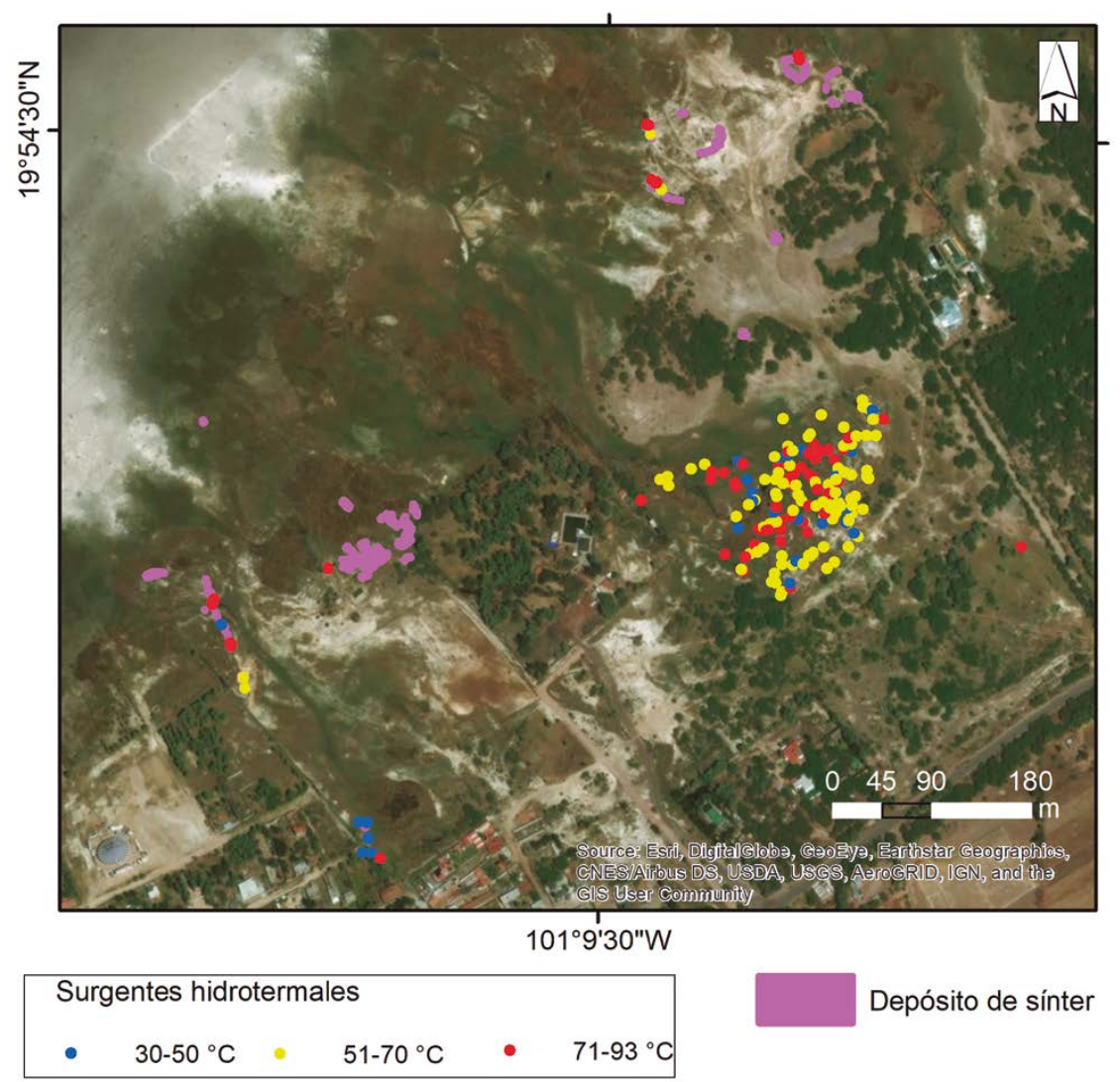

Figura 2. Área con surgentes termales $\left(32\right.$ y $\left.93^{\circ} \mathrm{C}\right)$ y depósitos hidrotermales a un costado de la comunidad de San Agustín del Maíz. 

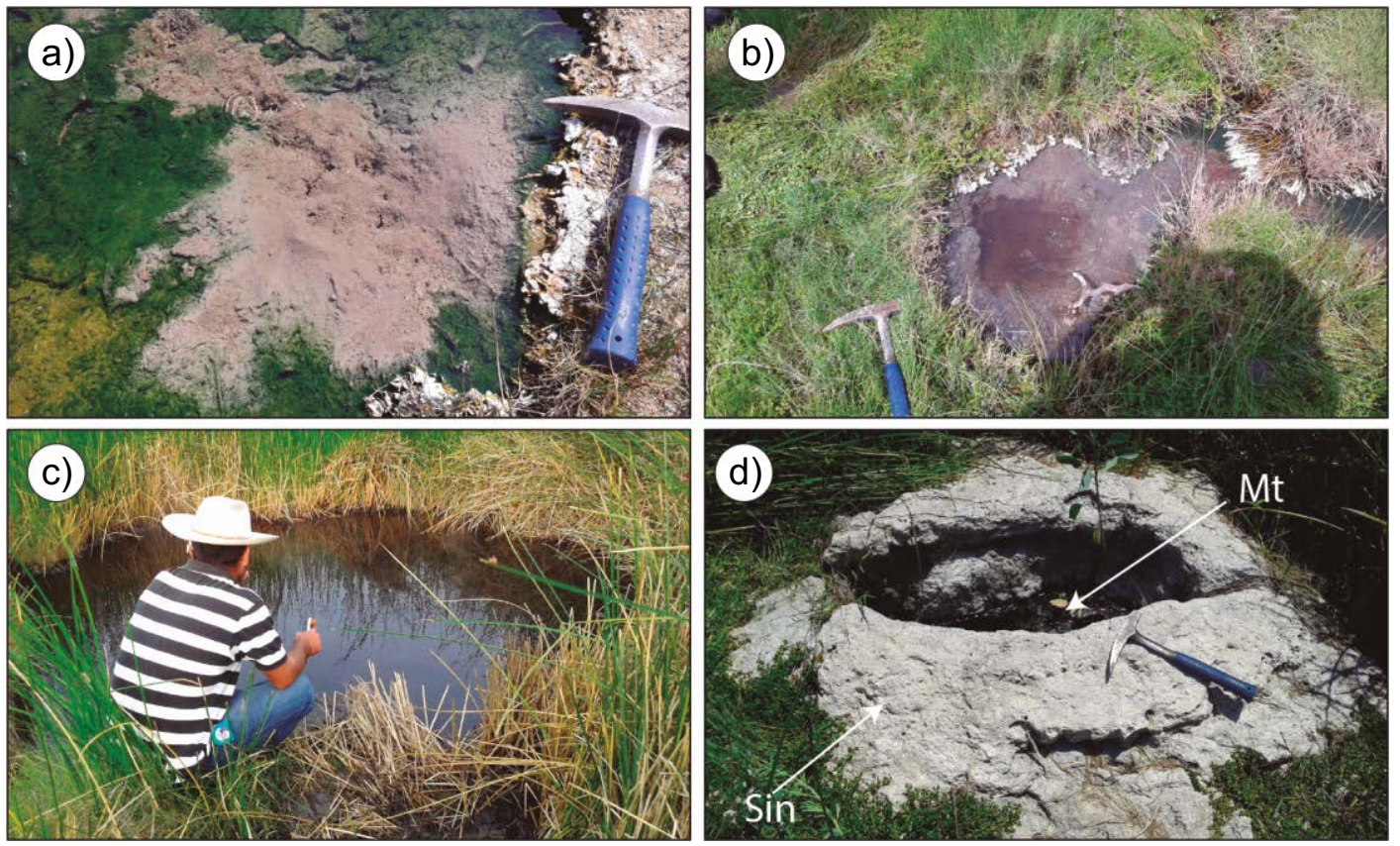

Figura 3. Manifestaciones hidrotermales; a) manantial termal; b) manantial hirviente; c) cráter de erupción hidrotermal; d) montículo de sínter (Sin) fracturado asociado a un manatial termal (Mt).

también depósitos de sínter (Figuras 2 y 3d) y brechas hidrotermales superficiales.

Los depósitos de sínter se pueden separar en dos tipos considerando el modo de deposición: depósitos bandeados asociados a fracturas (Figura 4) y depósitos sub-horizontales (Figura 5), siendo los primeros poco comunes. Los depósitos bandeados se encuentran asociados a fracturas que afectan a los depósitos sub-horizontales y son casi perpendiculares a la superficie (Figura 4a). La textura bandeada de estos depósitos alterna niveles vítreos y masivos con niveles porosos de pocos milímetros de espesor, y presenta coloraciones de entre café claro y gris (Figura 4b).

Por su parte, los depósitos sub-horizontales presentan estructuras de estratificación, además de que en estos se identificaron diferentes tipos de litofacies (Figura 5). Las litofacies más comunes son: vítreas de color negro (Figura 5a), semi-vítreas de color grisáceo (Figura 5b), semi-vítreas de color negro con impresiones de tallos de plantas (en algunos casos silicificados) y algunos intersticios (Figura 5c), semi- arenosa color grisáceo que presenta impresiones de tallos de plantas e intersticios (Figura 5d), facies ricas en tallos de plantas y sedimentos con mayor contenido de intersticios (Figura 5e), y facies ricas en sedimentos, fragmentos líticos y tallos de plantas. Cabe destacar, que el depósito vítreo fue explotado en tiempos pre-Hispánicos para generación de herramientas punzocortantes (Figura 5f). Estas litofacies mencionadas forman horizontes o estratos que van desde pocos milímetros formando una textura laminada, y llegan hasta los $18 \mathrm{~cm}$ de espesor formando una textura masiva.

De la misma forma, los depósitos hidrotermales presentan diferentes morfologías, siendo las principales: montículos, depósitos de fractura y terrazas (Figura 6). Los montículos presentan diámetros que van desde pocos centímetros hasta $20 \mathrm{~m}$, espesores de hasta $1.5 \mathrm{~m}$ y se observa que fueron formados a partir de una manantial termal central (Figura 6a). Los depósitos de fractura presentan espesores de hasta $30 \mathrm{~cm}$ y se forman por fluidos hidrotermales que emergen a partir de segmentos de fracturas que afectan o afectaron el terreno y que siguen
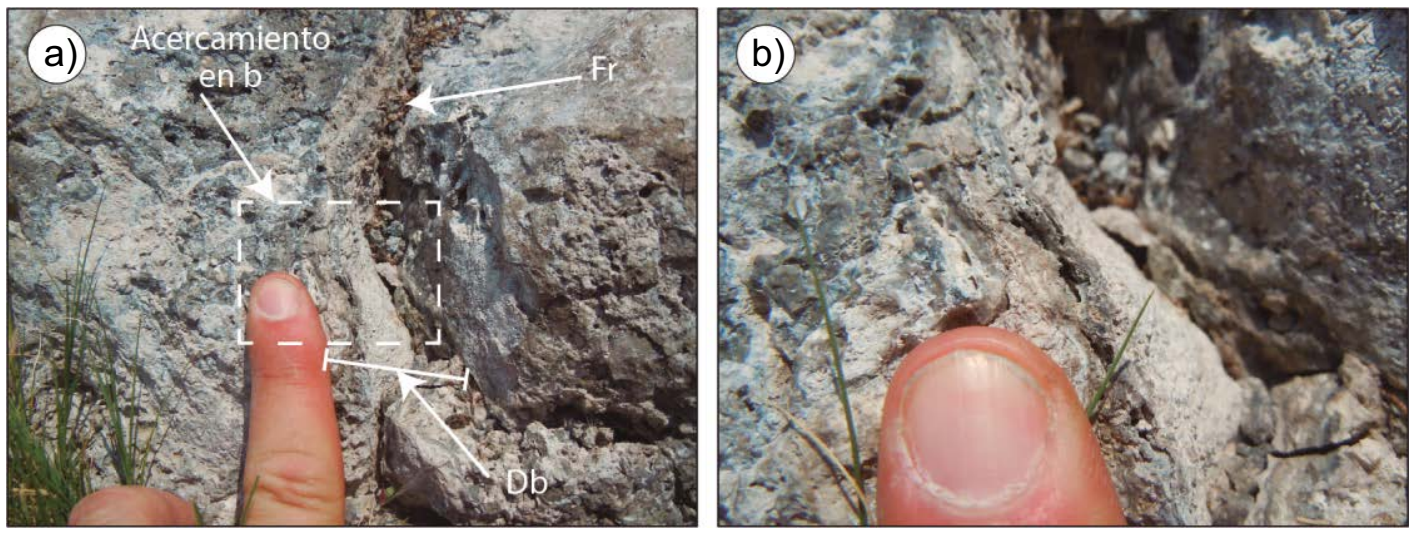

Figura 4. a-b) Deposito hidrotermal bandeado (Db) asociado a una fractura (Fr) que afecta un depósito hidrotermal sub-horizontal. 

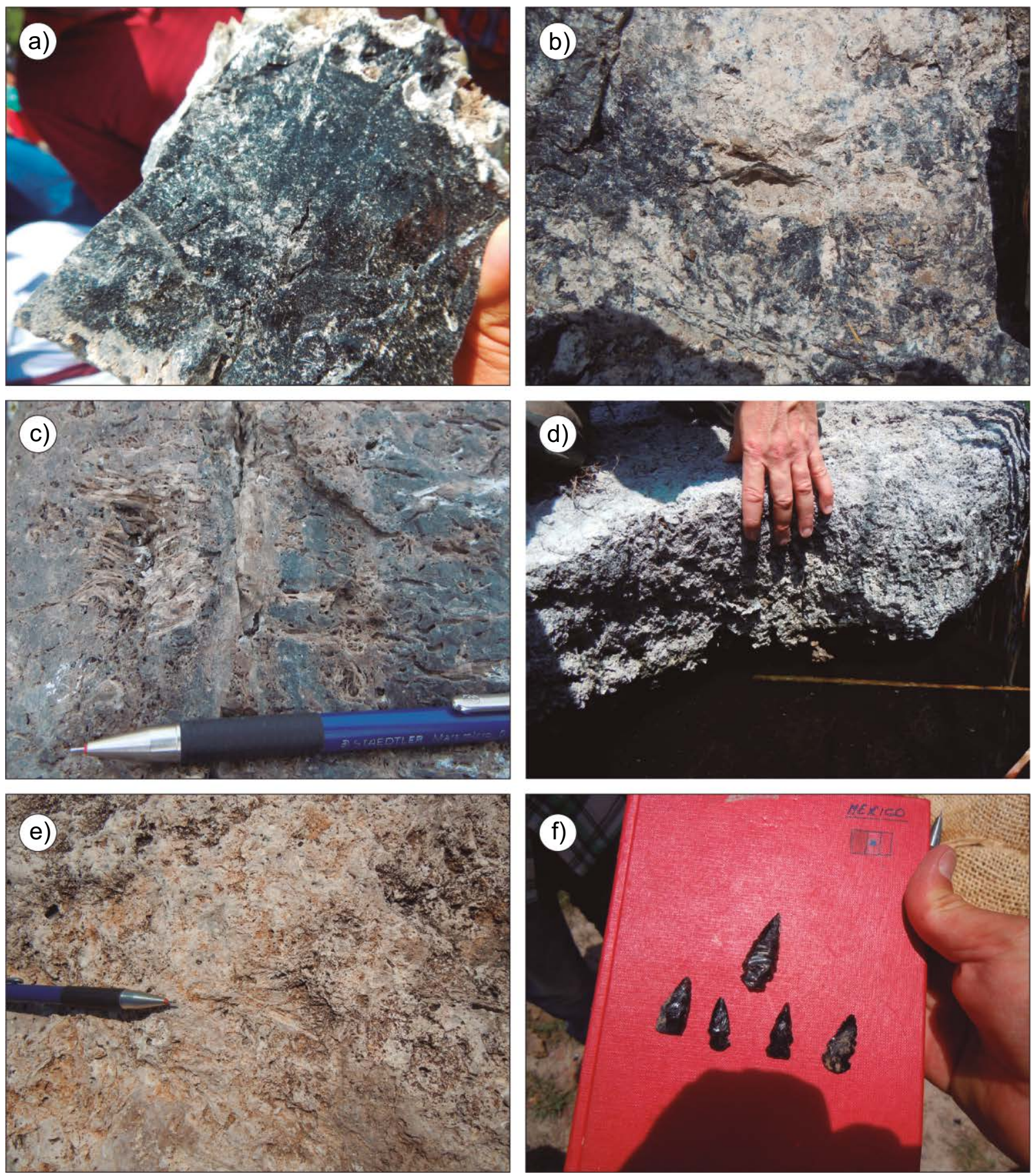

Figura 5. Litofacies de los depósitos hidrotermales sub- horizontales; a) vítrea; b) semi- vítrea; c) semi- vítrea con impresión de plantas; d) semi- arenosa con impresión de plantas; e) arenosa con impresión de plantas; f) puntas de flechas pre-Hispánicas hechas con el sínter vítreo.

dos rumbos preferenciales, SO-NE y NNO-SSE (Figura 6b). Por último, los depósitos de terrazas forman parches en el terreno y cubren áreas de hasta $130 \mathrm{~m}^{2}$, con espesores de hasta $50 \mathrm{~cm}$ y en algunos casos se observa que se formaron a partir de fisuras del terreno (Figura 6c).

También fueron identificadas litofacies de brechas hidrotermales que presentan tonalidades que van de café obscuro a café claro y las cuales no presentan estructuras sedimentarias (Figura 7a y 7b). En estas litofacies se identificaron tallos de plantas dispuestos caóticamente, líticos andesíticos que van de algunos $\mathrm{mm}$ hasta $15 \mathrm{~cm}$ de diámetro, poros de hasta $2 \mathrm{~cm}$ de diámetro, fragmentos de ópalo, fragmentos de cerámica prehispánica y algunos fragmentos de carbón (Figura 7c-7d). Todo lo anterior cementado por minerales hidrotermales.

Por otro lado, los cuatro análisis mineralógicos de DRX y espectroscopía infrarroja fueron realizados en muestras de sínter que presentan diferentes litofacies. La muestra Sam-1 presenta litofacies vítrea, la muestra Sam-2 litofacies semi-vítrea, la muestra Sam-3 corresponde al depósito de brecha y finalmente la muestra Sam-4 litofacies limo-arenosa. 

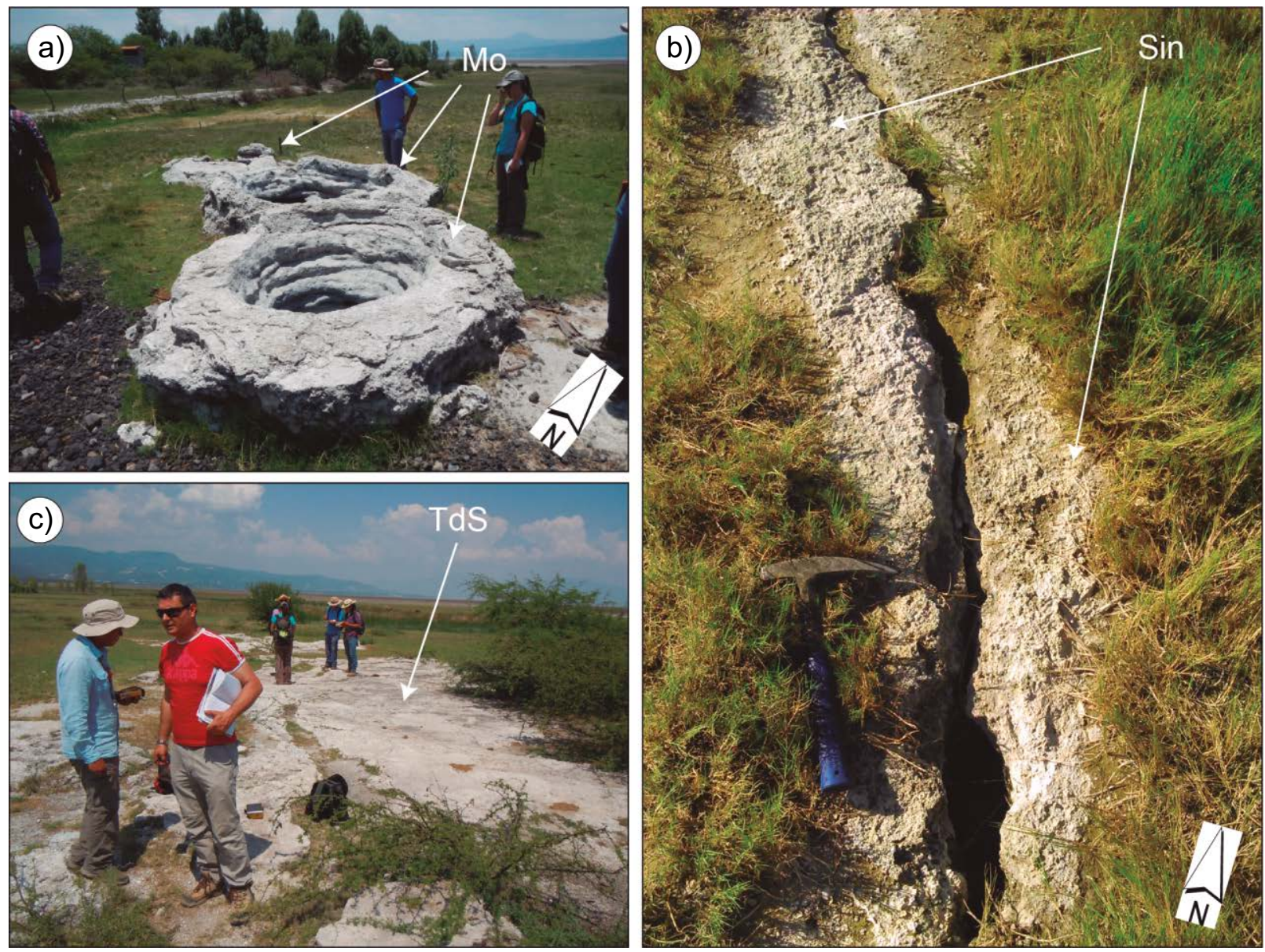

Figura 6. Morfologías de los depósitos hidrotermales; a) montículos (Mo) alineados en dirección NO-SE; b) depósitos de sínter (Sin) asociados a una fractura de dirección NNO-SSE; c) terrazas de sínter (TdS).

El constituyente mineralógico de la muestra Sam-1 contiene algunas características de fase mineral, tales como sílice libre cristalino polimorfo: ópalo- $\mathrm{CT}\left(\mathrm{SiO}_{2} \mathrm{n} \mathrm{H}_{2} \mathrm{O}\right)$. En los patrones de DRX del sínter investigado, la parte cristalina, está formada por cristobalita (ópaloC) y en algunos casos por la combinación de cristobalita y tridimita (ópalo-CT) (Figura 8). En particular, el ópalo-CT muestra un pico que corresponde a un valor $d$ con valores que van de 4.06 a $4.11 \AA$, mientras que el ópalo- $C$ se caracteriza por un pico entre 4.02 y $4.05 \AA$ y por la presencia de otros picos entre 3.13 y $2.84 \AA$ (e.g. Ostrooumov et al., 1999).

La composición mineral de las otras muestras de sínter estudiadas se caracteriza por la presencia de ópalo-CT acompañado de algunas fases mineralógicas típicas. En la muestra Sam-2, el ópalo se encuentra acompañado por cuarzo y clinoptilolita, en la muestra Sam-3 por cuarzo, estilbita, andesina y montmorillonita, y finalmente en la muestra Sam-4 por cuarzo, sanidina, clinoptilolita y montmorillonita (Figura 9).

Por su parte, los resultados de la espectroscopía infrarroja de las muestras estudiadas han sido comparados con los datos de DRX. Las bandas de absorción infrarroja fueron observadas en las regiones entre 4000 y $400 \mathrm{~cm}^{-1}$, las cuales son típicas para todas las variedades de ópalos micro- y no-cristalinos (tipo C, CT, y A). Los espectros infrarrojos de las muestras están caracterizados por una banda ancha de absorción entre 3700 y $2700 \mathrm{~cm}^{-1}$ (Figura 10), debido a la fundamental vibración de estiramiento del $\mathrm{OH}$ en la molécula de agua, así como la vibración de flexión del agua entre $\sim 1635-1648 \mathrm{~cm}^{-1}$ (Figura 10). Una banda ancha está presente alrededor de $3448-3458 \mathrm{~cm}^{-1}$ con un "hombro" alrededor de $3250 \mathrm{~cm}^{-1}$. Esta banda se considera generalmente relacionada al enlace de hidrógeno en la molécula de agua tal como en el agua líquida. Todas las muestras no cristalinas tienen de un hombro débil a un pico moderado en $950-960 \mathrm{~cm}^{-1}$, atribuible al enlace Si-O de los grupos $\mathrm{SiOH}$. En la región espectral entre 1500 y $400 \mathrm{~cm}^{-1}$ también se observan otras tres fuertes bandas en $\sim 1100 \mathrm{~cm}^{-1}, 790 \mathrm{~cm}^{-1}$, y $471 \mathrm{~cm}^{-1}$ donde son comunes todos los silicatos tetraédricamente coordinados y que son asignados al estiramiento por vibración del Si-O. En particular, las bandas en 1100 y $790 \mathrm{~cm}^{-1}$ son generalmente asignadas, respectivamente, al estiramiento asimétrico y simétrico del $\mathrm{Si}-\mathrm{O}-\mathrm{Si}$, donde en $470 \mathrm{~cm}^{-1}$ la banda es relacionada a la vibración de flexión del O-Si-O (Jones y Segnit, 1971; Farmer, 1974; Zarubin, 2001 y referencias en este; Fritsch et al., 2004; Brajkovic et al., 2007; Ostrooumov, 2007; Caucia et al., 2008; Adamo et al., 2010).

\section{DISCUSIÓN}

Asociados a la circulación y paleo-circulación de fluidos hidrotermales en SAM se identificaron depósitos hidrotermales superficiales, los cuales, desde el punto de vista de la deposición se separan en depósitos verticales-bandeados y los depósitos sub-horizontales (estratificados). Los primeros, debido a su relación con las fracturas, su geometría vertical y textura bandeada pueden correlacionarse con vetas (e.g. Cox et al., 2001) y con las litofacies bandeadas en los travertinos (Gandin y Capezzuoli, 2014), por lo tanto, pueden asociarse a la 
deposición en los conductos a través de los cuales los fluidos llegan a la superficie (Figura 4d). Los depósitos sub-horizontales por su parte, son depósitos que se formaron a partir de los fluidos que llegaron a la superficie, permitiendo la formación de estructuras de estratificación.

Las litofacies identificadas en los depósitos estratificados, a excepción de la litofacies de brecha que se asocian a erupciones hidrotermales (e.g. Browne y Lawless, 2001; Canet et al., 2015), se pueden enmarcar en un rango con dos miembros finales, donde por un lado se encuentra el depósito vítreo y por el otro lado el depósito limo-arenoso (Figura 11). A partir de la parte vítrea se inicia un aumento transicional hacia la parte limo-arenosa en el contenido de plantas, sedimentos, poros y líticos, además de que disminuye el contenido de sílice (Figura 11). De hecho, este cambio transicional de las litofacies es similar al identificado en los depósitos de sínter de sílice del campo geotérmico de Ixtlán de los Hervores (Michoacán) (Olvera-García et al., 2020b), además de que en otros campos geotérmicos como San Bartolomé de los Baños en Guanajuato (Canet et al., 2019), Araró, Los Azufres y Puruándiro en Michoacán fueron identificadas litofacies similares a las de SAM.

El contenido de ópalo ( $\mathrm{C}$ y CT) en los depósitos analizados permite clasificarlos como sínter de sílice (Campbell et al., 2015), sugiriendo la presencia de fluidos geotérmicos que se han equilibrado con las rocas en el subsuelo en temperaturas $>175^{\circ} \mathrm{C}$ (e.g. Fournier y Rowe, 1966). Esta temperatura concuerda con las temperaturas determinas por diferentes geotermómetros en SAM, las cuales van de los 138 a los $197^{\circ} \mathrm{C}$ (Segovia et al., 2005). La mineralogía identificada en los depó- sitos estratificados se puede correlacionar con las litofacies; las facies vítreas están formadas solamente por ópalo, mientras que al acercarse a la parte limo-arenosa se identifica mayor diversidad mineralógica.

El sínter puro presenta las características del depósito conocido como geiserita (e.g. Lynne, 2012; Campbell et al., 2015), el cual es un depósito de sínter proximal a la surgente, formado por fluidos hidrotermales con temperaturas en la superficie de entre 70 y $100{ }^{\circ} \mathrm{C}$ (e.g. Pentecost, 2005; White et al., 1964; Campbell et al., 2015), representando un ambiente en el que no pueden sobrevivir las plantas. El resto de las litofacies, donde se encuentran los tallos de plantas impresos y/u opalizados, sugieren un ambiente donde la temperatura del agua $\left(<70^{\circ} \mathrm{C}\right)$ permitió el crecimiento de estas (e.g. Lynne, 2012; Campbell et al., 2015). Estas plantas favorecen la acumulación de sedimentos y, por ende, la formación de sínter enriquecidos con dichos sedimentos. Por otro lado, la formación de niveles o estratos milimétricos que da lugar a una textura laminada indica una tasa de deposición lenta, mientras que las texturas más masivas indican una alta tasa de deposición (e.g. Lynne, 2012).

En este sentido se considera que la textura, las litofacies y la composición mineralógica de los depósitos de sínter de SAM representan herramientas para identificar escenarios con mayor circulación de fluidos y mayores temperaturas. En particular, las zonas con facies masivas de geiserita sugerirían escenarios favorables para la intensa circulación de fluidos y temperaturas superiores de $70^{\circ} \mathrm{C}$. Este análisis puede ser muy útil durante las primeras etapas de exploración
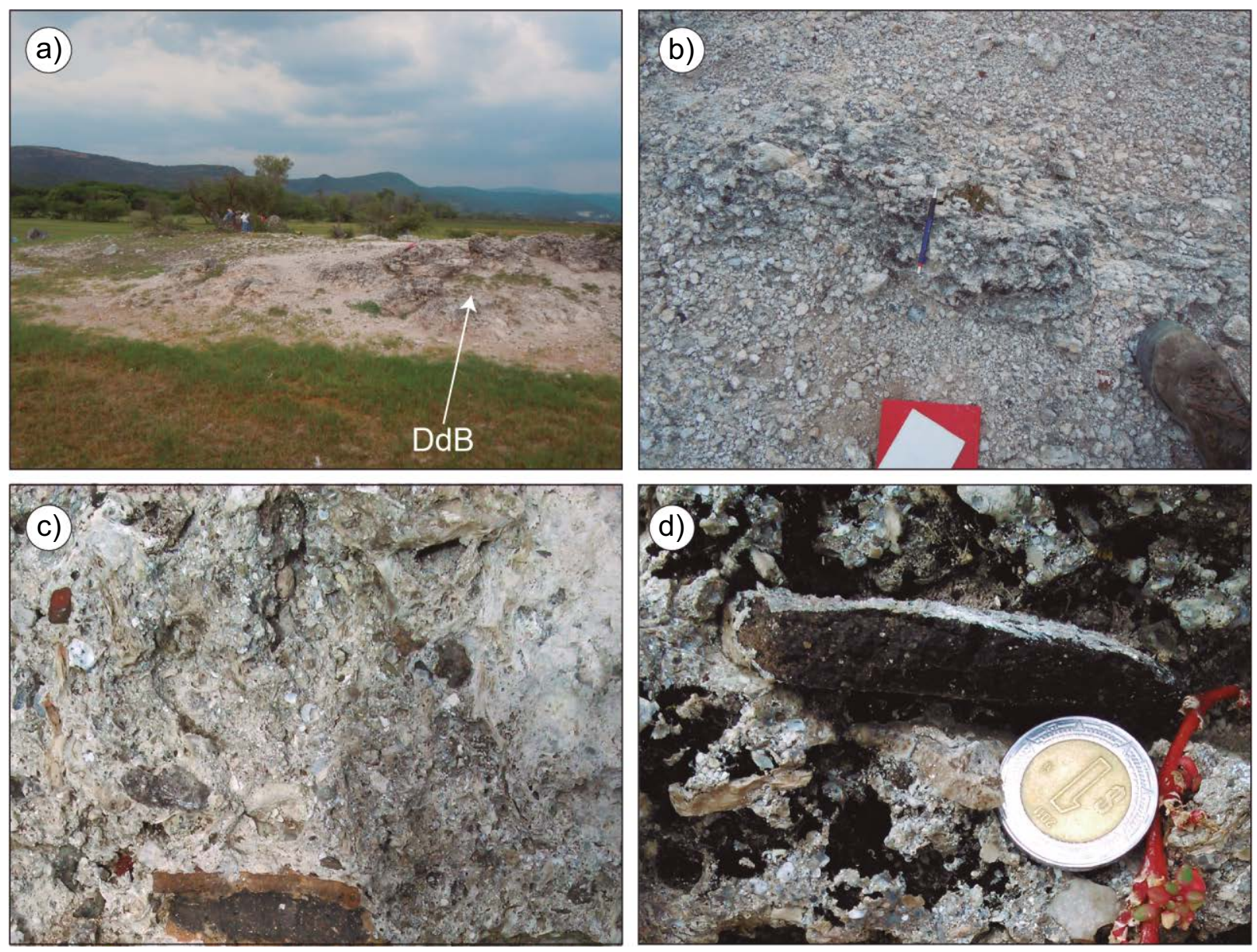

Figura 7. Depósitos de brechas hidrotermales superficiales; a-b) montículos de depósitos de brecha (DdB); c-d) depósitos de brecha con contenido de líticos, cerámica pre-Hispánica y fragmentos de ópalo. 


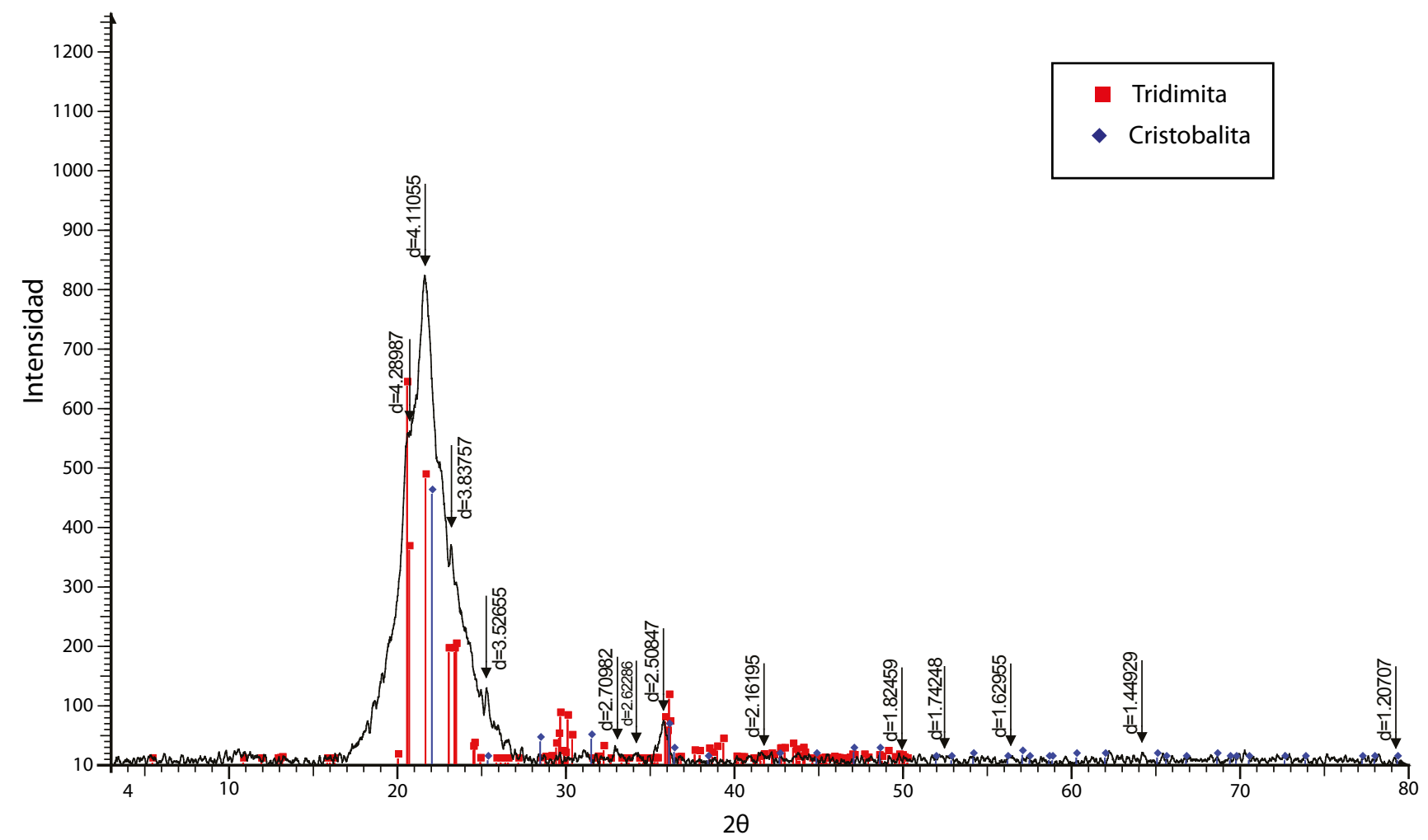

Figura 8. Difractográma de la muestra Sam-1, evidenciando un pico máximo del valor d en 4.11 Å y otros picos entre 3.5 y 2.7 Å, asociados a ópalos C y CT.

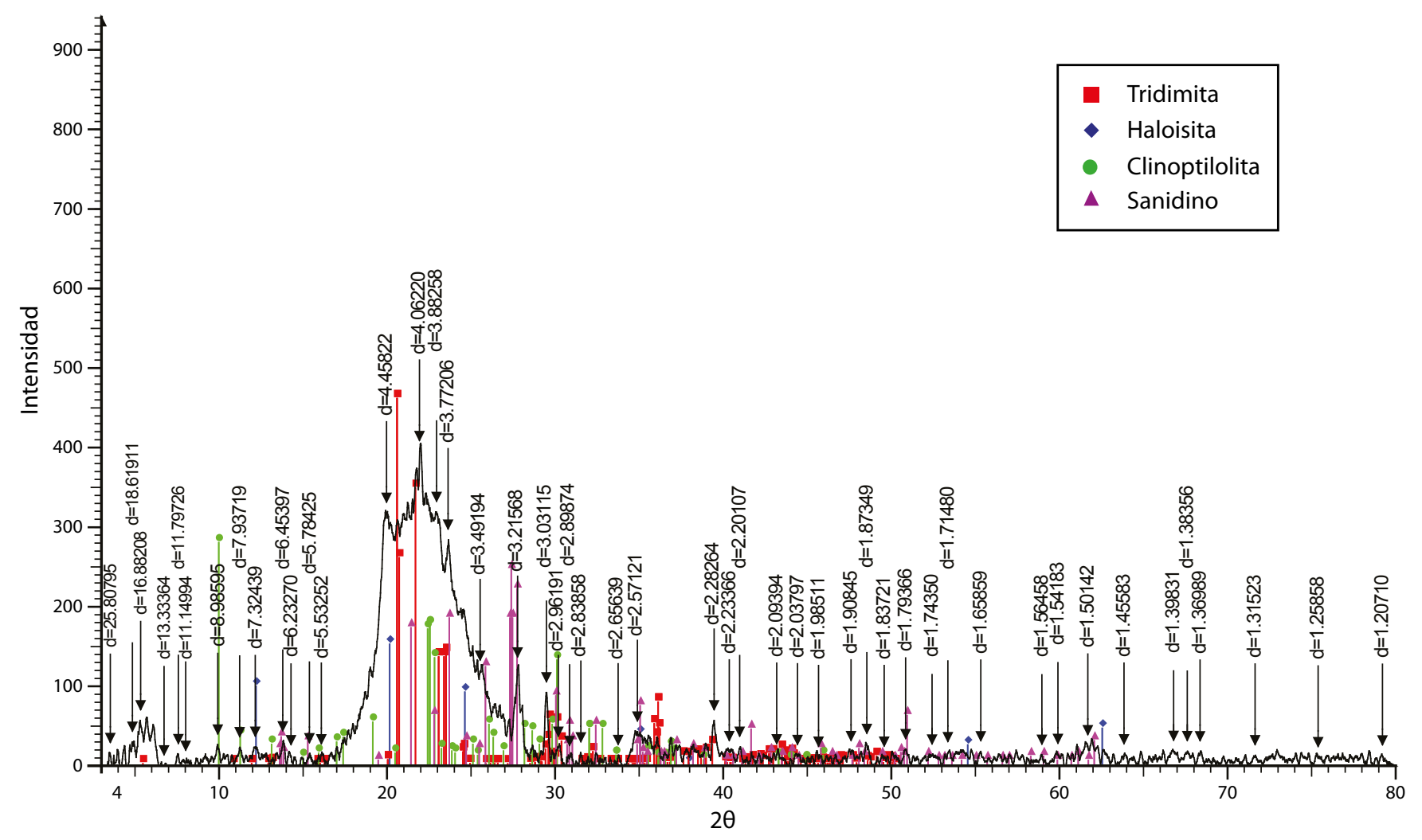

Figura 9. Difractográma de la muestra Sam-4, evidenciando picos del valor d asociados a ópalo-CT, halloysita, clinoptilolita y sanidino. 


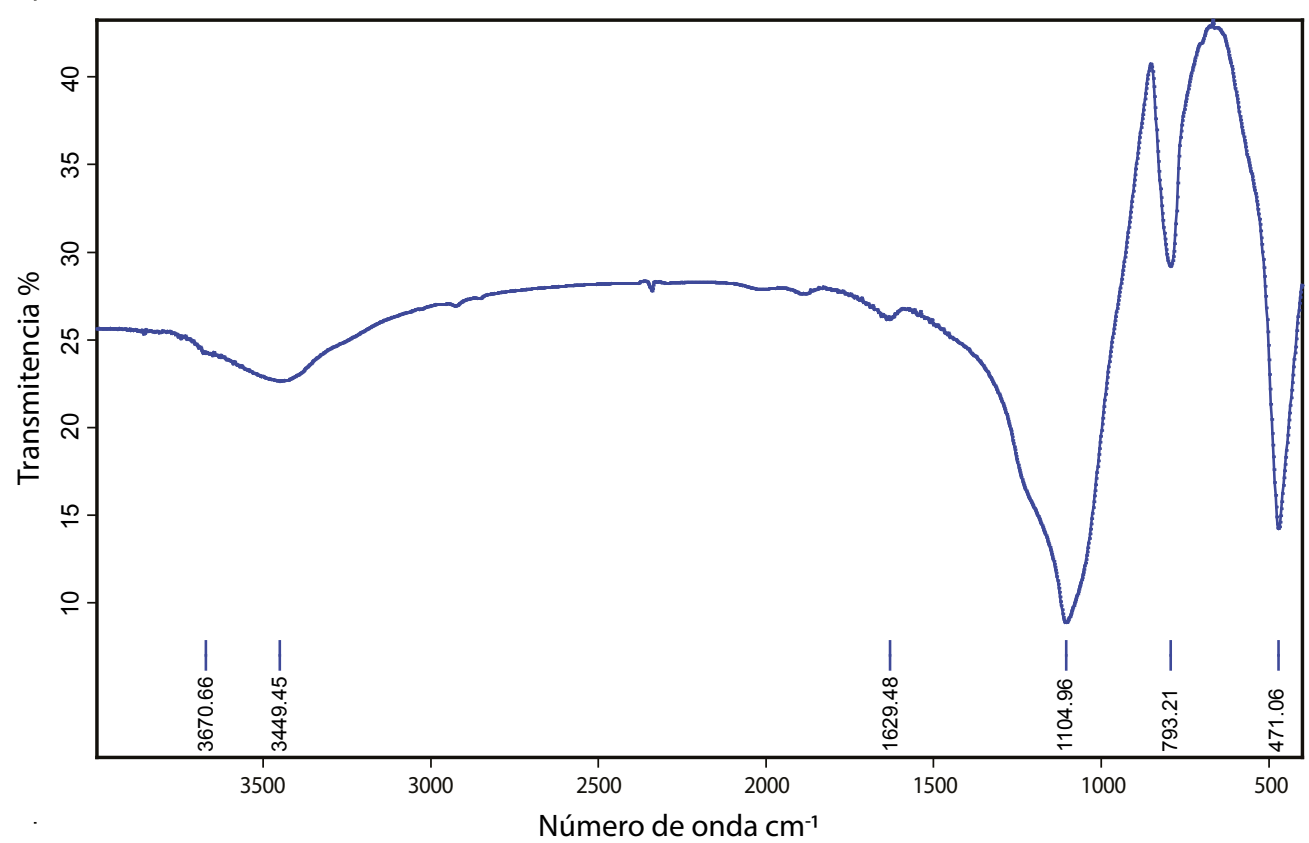

Figura 10. Gráfico representando los resultados de la espectroscopía infrarroja de la Sam-1. Las bandas de absorción infrarroja se encuentran entre las regiones 4000 y $400 \mathrm{~cm}^{-1}$, las cuales son típicas para todas las variedades de ópalos micro- y no-cristalinos (C, CT, y A). Los espectros infrarrojos de las muestras son caracterizados por una ancha banda de absorción entre 3700 y $2700 \mathrm{~cm}^{-1}$, debido a la fundamental vibración de estiramiento del OH en la molécula de agua, así como la vibración de flexión del agua entre $\sim 1635-1648 \mathrm{~cm}^{-1}$.

geotérmica y puede ser usado en zonas geotérmicas con escenarios geológicos similares al de SAM, como pueden ser otras zonas dentro del sector central del CVTM.

Se ha observado que escenarios estructurales favorables permiten el ascenso de fluidos geotérmicos a la superficie con temperaturas entre los 70 y $100^{\circ} \mathrm{C}$ (e.g. Taillefer et al., 2017; Olvera-García et al., 2020a). Según Olvera-García et al. (2020a), en la zona geotérmica de SAM, la intersección de fallas (OSO-ENE y NNO-SSE) da lugar a un gran volumen de roca deformada y a la formación de pipe-like structures (e.g. Rowland y Sibson, 2004) que crean canales de circulación vertical, formando en superficie zonas puntuales con mayor circulación de fluidos y con temperaturas superiores a los $70^{\circ} \mathrm{C}$ (Figura 12). A partir de los pipe-like structures, los fluidos también circularían horizontal y sub- verticalmente a lo largo de las estructuras, sin embargo, a una velocidad menor, teniendo más tiempo de interactuar con el nivel freático y enfriándose mientras llegan a la superficie (Figura 12). Otros escenarios geológicos que es sabido que permiten la alta circulación vertical de fluidos geotérmicos son terminación de fallas y rampas de relevo (e.g. Faulds et al., 2011; Curewitz y Karson, 1997).

Por otro lado, tomando en cuenta la morfología de los depósitos de sínter de la zona de SAM, se considera que los montículos se forman en escenarios donde la estructura (e.g. zona de falla) que controla el ascenso de fluidos a la superficie se encuentra enterrada por varios metros por depósitos no consolidados (e.g. sedimentos lacustres), mientras que, cuando se forma la fisuras en la superficie, el lecho rocoso afectado por las estructuras que controlan los fluidos se encuentra muy

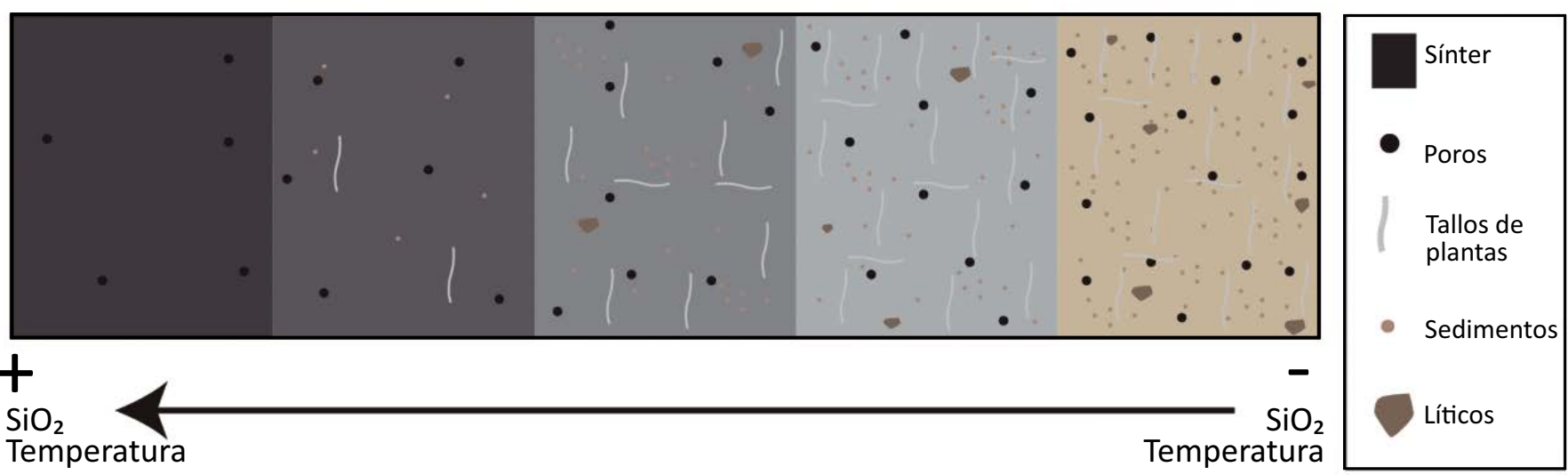

Figura 11. Gráfico que representa conceptualmente las diferentes litofacies de los depósitos de sínter de sílice, donde, por un lado se encuentra las litofacies vítreas con mayor contenido de sílice y las cuales se ha formado a partir de fluidos hidrotermales con temperaturas entre 70 y $100^{\circ} \mathrm{C}$, mientras que del otro lado se encuentra las litofacies arenosas con menor contenido de sílice y formado a partir de fluidos con temperaturas de entre 70 y $30^{\circ} \mathrm{C}$. 


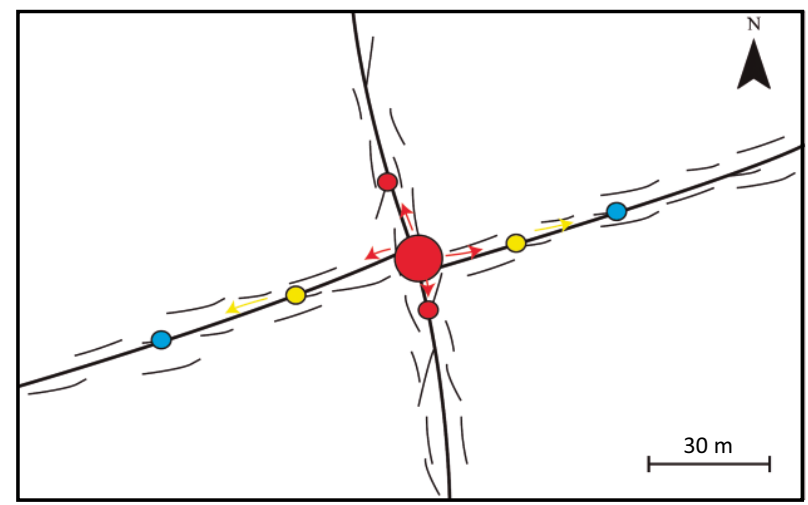

Figura 12. Esquema que representa el escenario estructural de la zona geotérmica de SAM, donde la intersección de la zona falla normal OSO-ENE con la zona de falla lateral NNO-SSE forma una pipe-like structure que conduce verticalmente fluidos profundos. Esta intersección presenta el mejor escenario para una alta circulación de fluidos con temperaturas mayores a $70^{\circ} \mathrm{C}$ (círculo rojo) y por ende para la formación de depósitos hidrotermales con facies de Geiserita con facies masivas. A partir de la intersección los fluidos migran lateralmente a través de la falla principal, interactuando con el nivel freático y enfriándose poco a poco, donde finalmente ascienden a la superficie en la intersección con fracturas secundarias (círculos amarillo y azul).

somero o inclusive aflorando en superficie (e.g. Hancock et al., 1999; Pentecost, 2005) (Figura 13). fluidos a la superficie y los segundos se forman a partir de los fluidos que llegaron a la superficie. Por su parte, las litofacies que forman los depósitos estratificados se pueden agrupar en un rango con dos miembros finales, donde por un lado se encuentra un depósito con aspecto vítreo formado solo por ópalo y por el otro, un depósito con aspecto limo-arenoso y formado por ópalo en conjunto con otras fases mineralógicas asociadas a sedimentos.

Las litofacies de los depósitos de sínter son controladas principalmente por la tasa de deposición, la temperatura del agua y la distancia de depósito respecto de la fuente hidrotermal. En particular, los depósitos de geiserita con facies masivas sugieren ambientes hidrotermales con temperaturas superiores a los $70^{\circ} \mathrm{C}$ y donde ha existido mayor circulación hidrotermal en la superficie. Estos ambientes pueden coincidir con escenarios estructurales conocidos por la alta circulación vertical de fluidos geotérmicos como en las intersecciones de fallas, terminación de fallas y rampas de relevo. En este sentido, los depósitos hidrotermales con facies de geiserita son una herramienta para identificar las áreas donde existió mayor circulación hidrotermal con mayores temperaturas en zonas geotérmicas con escenarios geológicos similares al de SAM.

Finalmente, se considera que el espesor de sedimentos no consolidados recubriendo las estructuras (fracturas) por las que ascienden los fluidos geotérmicos a la superficie, determina la geometría de los depósitos de sínter. En el caso de que el espesor de los sedimentos no consolidados sea escaso y que el lecho rocoso se encuentre cerca de la superficie, la morfología será de tipo fractura, mientras que cuando el espesor de los sedimentos no consolidados sea mayor, se formaran los montículos.

\section{CONCLUSIONES}

En la zona geotérmica de SAM, los depósitos de sínter son de dos tipos; verticales-bandeados y estratificados, donde los primeros se asocian a deposición en los conductos a través de los cuales llegan los

\section{AGRADECIMIENTOS}

Queremos dedicar este manuscrito a la memoria del Dr. Víctor Hugo Garduño Monroy, entrañable maestro, compañero, y amigo

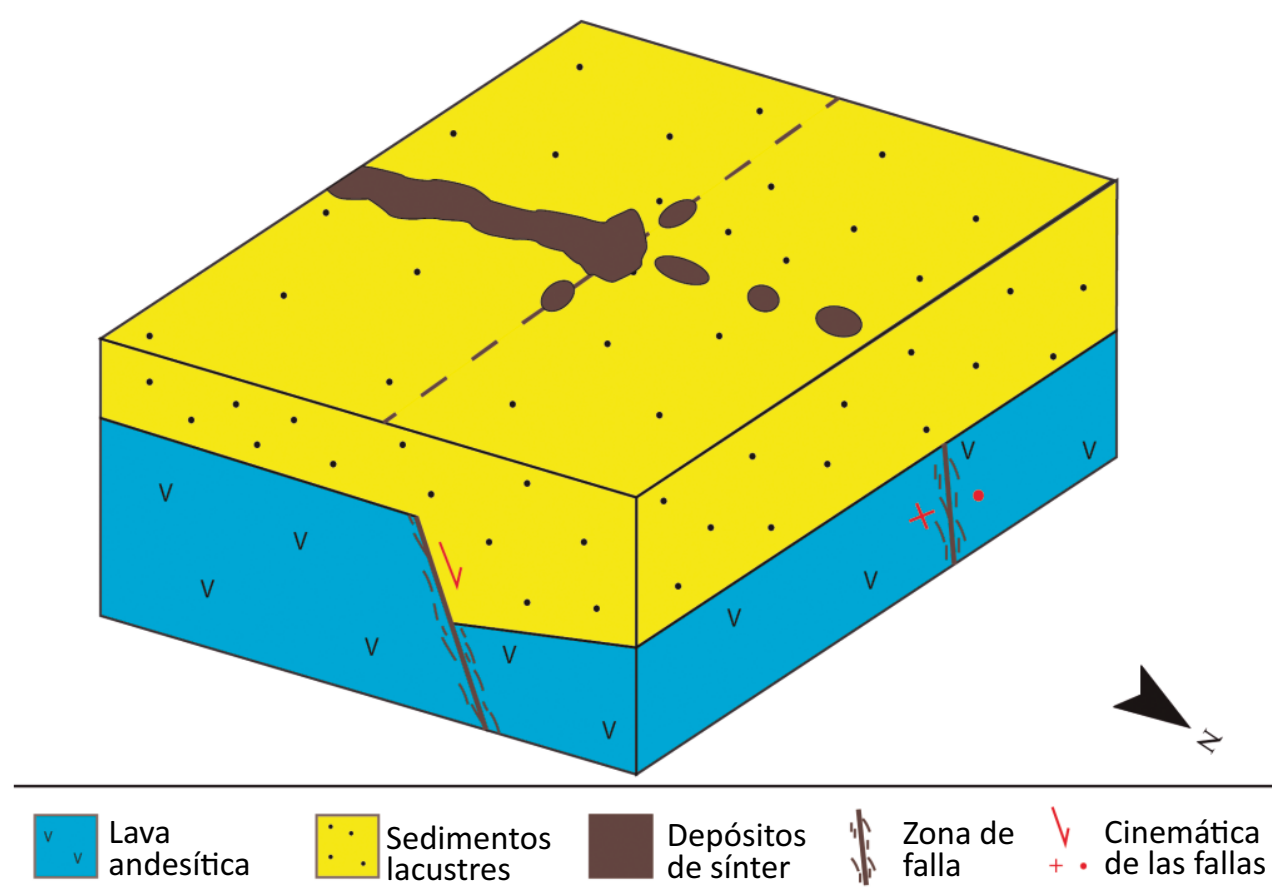

Figura 13. Esquema que representa cómo una zona de falla que controló la circulación de fluidos es cubierta por un pequeño espesor de sedimentos no consolidados dando lugar a la formación de una fisura, y cuando el espesor de sedimentos no consolidados es mayor, da lugar a la formación de montículos alineados. 
quien se nos adelantó en el camino: te extrañamos. Este trabajo fue financiado por el proyecto-17 (P-17) del CEMIE-Geo. Queremos agradecer a los integrantes del P-17 del CEMIE-Geo por su apoyo durante muchas de las visitas a campo. E.O.G. agradece al CONACYT por las becas de maestría y doctorado otorgadas, y durante las cuales este trabajo de investigación fue realizado. Finalmente, agradecemos a los editores Lucia Capra y Ángel Francisco Nieto-Samaniego, así como a los revisores del manuscrito Carles Canet y uno anónimo por sus comentarios y sugerencias que ayudaron a mejorar dicho manuscrito.

\section{REFERENCIAS}

Adamo, I., Ghisoli, C., Caucia, F., 2010, A contribution to the study of FT-IR spectra of opals: Neues Jarbuch für Mineralogie Abhandlungen, 187, 63-68.

Boudreau, A.E., Lynne, B.Y., 2012, The growth of siliceous sinter deposits around high temperature eruptive hot springs: Journal of Volcanology and Geothermal Research, 247-248, 1-8.

Brajkovic, A., Rolandi, V., Vignola, P., Grizzetti, R., 2007, Blue and pink opals from Acari, Peru - their optical, structural and spectroscopic features: The Australian Gemmologist, 23, 3-15.

Brogi, A., Capezzuoli, E., Buracchi, E., Branca, M., 2012, Tectonic control on travertine and calcareous tufa deposition in a low-temperature geothermal system (Sarteano, Central Italy): Journal of the Geological Society, 169, 461-476.

Browne, P.R.L., Lawless, J.V., 2001, Characteristics of hydrothermal eruptions with examples from New Zealand and elsewhere: Earth-Science Reviews, 52, 299-331.

Campbell, K.A., Guido, D.M., Gautret, P., Foucher, F., Ramboz, C., Westall, F., 2015, Geyserite in hot-spring siliceous sinter: Window on Earth's hottest terrestrial (paleo) environment and its extreme life: Earth-Science Reviews, 148, 44-64.

Canet, C., Hernández-Cruz, B., Jiménez-Franco, A., Pi, T., Peláez, B., Villanueva-Estrada, R.E., Alfonso, P., González-Partida, E., Salinas, S., 2015, Combining ammonium mapping and short-wave infrared (SWIR) reflectance spectroscopy to constrain a model of hydrothermal alteration for the Acoculco geothermal zone, Eastern Mexico: Geothermics, 53, 154-165.

Canet, C., Rodríguez-Díaz, A.A., Bernal, I.D., Pi, T., Sánchez-Córdova, M.M., Núñez-Useche, F., Villanueva-Estrada, R., Molina, G., Reich, M., Peláez, B., Jiménez Salgado, E., González-Partida, E., Sandoval Medina, F., CarrilloSánchez, C.B., 2019, Consideraciones sobre el sistema geotérmico de San Bartolomé de los Baños, Guanajuato (México), desde un análisis de la alteración hidrotermal y las inclusiones fluidas: Geofísica Internacional, 58(3), 229-246.

Caucia, F., Ghisoli, C., Adamo, I., Bocchio, R., 2008, Opals-C, opals-CT and opals-T from Acari, Peru: X-ray powder diffraction analysis and IR spectroscopic investigation of new samples showing two different typologies of lustre: The Australian Gemmologist, 23, 266-271.

Cox, S. F., Knackstedt, M. A., Braun, J., 2001, Principles of structural control on permeability and fluid flow in hydrothermal system: Reviews in Economic Geology, 14, 1-24.

Cunneen, R., Sillitoe, R.H., 1989, Paleozoic hot spring sinter in the Drummond Basin, Queensland, Australia: Economic Geology, 84, 135-142.

Curewitz, D., Karson, J.A., 1997, Structural settings of hydrothermal outflow: Fracture permeability maintained by fault propagation and interaction: Journal of Volcanology and Geothermal Research, 79, 149-168.

Demant, A., 1978, Características del Eje Neovolcánico Transmexicano y sus problemas de interpretación: Revista Mexicana de Ciencias Geológicas, 2, 172-187.

Ego, F., Ansan, V., 2002, Why is the Central Trans-Mexican Volcanic Belt $\left(102^{\circ}-99^{\circ} \mathrm{W}\right)$ in transtensive deformation?: Tectonophysics, 359, 189-208

Farmer, V.C., 1974. The infrared spectra of minerals: London, UK, Mineralogical Society, $539 \mathrm{pp}$.

Faulds, J.E., Coolbaugh, M.F., Hinz, N.H., Cashman, P.H., Kratt, C., Dering, G., Edwards, J., Mayhew, B., McLachlan, H., 2011, Assessment of favorable structural settings of geothermal systems in the Great Basin, western USA: Geothermal Resources Council Transactions, 35, 777-784.
Ferrari, L., Orozco-Esquivel, T., Manea, V., Manea, M., 2012, The dynamic history of the Trans-Mexican Volcanic Belt and the Mexico subduction zone: Tectonophysics, 522-523, 122-149.

Fournier, R.O., Rowe, J.J., 1966, Estimation of underground temperatures from the silica content of water from hot springs and steam Wells: American Journal of Science, 264, 685-697.

Fritsch, E., Gaillou E., Ostrooumov, M., Rondeau, B., Devouard, B., Barreau, A., 2004, Relationship between nanostructure and optical absorption in fibrous pink opals from Mexico and Peru: European Journal of Mineralogy, $16,743-752$.

Gandin, A., Capezzuoli, E., 2014, Travertine: Distinctive depositional fabrics of carbonates from thermal spring systems: Sedimentology, 61, 264-290.

Gómez-Tuena, A., Orozco-Esquivel, T., Ferrari, L., 2007, Igneous petrogenesis of the Trans-Mexican Volcanic Belt, en Alaniz-Álvarez, S.A., NietoSamaniego, Á.F. (eds.), Geology of México: Celebrating the Centenary of the Geological Society of México: Geological Society of America, Special Paper 422, 1-53.

Gómez-Vasconcelos, M.G., Garduño-Monroy, V.H., Macías, J.L., Layer, P.W., Benowitz, J.A., 2015, The Sierra de Mil Cumbres, Michoacán, México: transitional volcanism between the Sierra Madre Occidental and the Trans-Mexican Volcanic Belt: Journal of Volcanology and Geothermal Research, 15, 128-147.

Guido, D.M., Campbell, K.A., 2011, Jurassic hot spring deposits of the DeseadoMassif (Patagonia, Argentina): characteristics and controls on regional distribution: Journal of Volcanology and Geothermal Research, 203, 35-47.

Hancock, P.L., Chalmers, R.M.L., Altunel, E., Çakir, Z., 1999, Travitonics: using travertines in active fault studies: Journal of Structural Geology, 21, 903-916.

Herdianita, N., Browne, P., Rodgers, K., Campbell, K., 2000, Mineralogical and textural changes accompanying ageing of silica sinter: Mineralium Deposita, 35, 48-62.

Israde-Alcantara, I., 1997, Neogene diatoms of Cuitzeo Lake, central sector of the Trans-Mexican Volcanic Belt and their relationship with the volcanotectonic evolution: Quaternary International, 43-44, 137-143.

Israde-Alcantara, I., Garduño-Monroy, V.H., 1999, Lacustrine record in a volcanic intra arc setting: the evolution of the Late Neogene Cuitzeo basin system (central-western Mexico, Michoacán): Palaeogeography, Palaeoclimatology, Palaeoecology, 151, 209-227.

Johnson, E.R., Delgado, H., Manea, V.C., Kent, A.J.R., Bindeman, I.N., Donegan, C.S., 2009, Subduction-related volatile recycling and magma generation beneath Central Mexico: insights from melt inclusions, oxygen isotopes and geodynamic models: Journal of Petrology, 50(9), 1729-1764.

Jones, J.B., Segnit, E.R., 1971, The nature of opal. Part 1: Nomenclature and constituent phases: Journal of Geological Society of Australia, 8, 57- 68.

Lynne, B.Y., 2012, Mapping vent to distal-apron hot spring paleo-flow pathways using siliceous sinter architecture: Geothermics, 43, 3-24.

Olvera-García, E., Garduño-Monroy, V.H., Liotta, L., Brogi, A., BermejoSantoyo, G., Guevara-Alday, J.A., 2020a, Neogene-Quaternary normal and transfer faults controlling deep-seated geothermal systems: The case of San Agustín del Maíz (central Trans-Mexican Volcanic Belt, México): Geothermics, 86, 101791.

Olvera-García, E., Garduño-Monroy, V.H., Ostrooumov, M., Gaspar-Patarroyo, T.L., Nájera-Blas, S.M., 2020b, Structural control on hydrothermal upwelling in the Ixtlán de los Hervores geothermal area, Mexico: Journal of Volcanology and Geothermal Research, 339, 106888.

Ostrooumov, M., 2007, A Raman, infrared and XRD analysis of the instability in volcanic opals from Mexico. Spectrochimica Acta Part A: Molecular and Biomolecular Spectroscopy, 68(4), 1070-1076.

Ostrooumov, M., Fritsch, E., Lasnier, B., Lefrant, S., 1999, Spectres Raman des opales: Aspect diagnostique et aide a la classification: European Journal of Mineralogy, 11, 899-908.

Pasquaré, G., Ferrari, L., Garduño-Monroy, V.H., Tibaldi, A., Vezzoli, L., 1991, Geologic Map of the Central Sector of the Mexican Volcanic Belt, States of Guanajuato and Michoacan: Geological Society of America, Map and Chart Series, MCH072, 1 Map and Text, 22.

Pasquaré, G., Garduño, V.H., Tibaldi, A., Ferrari, M., 1988, Stress pattern evolution in the central sector of the Mexican Volcanic Belt: Tectonophysics, $146,353-364$. 
Pentecost, A., 2005, Travertine: Berlin, Springer-Verlag, 445 pp.

Renaut, R.W., Jones, B., 2003, Sedimentology of hot spring systems: Canadian Journal of Earth Sciences, 40, 1439-1442.

Rowland, J.V., Sibson, R.H., 2004, Structural controls on hydrothermal flow in a segmented rift system, Taupo Volcanic Zone, New Zealand: Geofluids, $4,259-283$.

Segovia, N., Barragan, R.M., Tello, E., Alfaro, R., Mena, M., 2005, Geochemical Characteristics and 222Rn Measurements at Cuitzeo Basin (Mexico) Thermal Springs and Artesian Wells, en Proceedings World Geothermal Congress 2005: Antalya, Turkey, 24-29 April 2005.

Suter, M., Aguirre, G., Siebe, C., Quintero, O., Komorowski, J.C., 1991, Volcanism and active faulting in the central part of the trans-Mexican Volcanic Belt, Mexico, en Walawender, M.J., Hanan, B.B. (eds.), Geological Excursions in Southern California and Mexico. Guidebook: Boulder, Annual Meeting of the Geological Society of America, 224-243.

Suter, M., Lopéz-Martínez, M., Quintero-Legorreta, O., Carrillo-Marinez, M., 2001. Quaternary intra-arc extension in the central Trans-Mexican volcanic belt: The Geological society of America Bulletin, 113, 693-703.

Taillefer, A., Soliva, R., Guillou-Frottier, L., Le Goff, E., Martin, G., Seranne, M., 2017, Fault-Related Controls on Upward Hydrothermal Flow: An Integrated Geological Study of the Têt Fault System, Eastern Pyrénées (France): Geofluids, 19, https://doi.org/10.1155/2017/8190109.
Walter, M.R., Des Marais, D., Farmer, J.D., Hinman, N.W., 1996, Lithofacies and biofacies of mid-Paleozoic thermal spring deposits in the Drummond Basin, Queensland, Australia: Palaios, 11, 497-518.

White, D.E., Thompson, G.A., Sandberg, C.H., 1964, Rocks, structure, and geologic history of Steamboat Springs thermal area, Washoe County, Nevada: U.S. Geological Survey Professional Paper 458-B, 63 pp.

Wohletz, K., Heiken, G., 1992, Volcanology and geothermal energy: Berkeley, CA, University of California Press, $432 \mathrm{pp}$.

Zarubin, D.P., 2001, The two-component bands at about 4500 and $800 \mathrm{~cm}^{-1}$ in infrared spectra of hydroxyl-containing silicas. Interpretation in terms of Fermi resonance: Journal of non-Crystalline Solids, 286, 80-88.

Manuscrito recibido: junio 17, 2020

Manuscrito corregido recibido: septiembre 11, 2020

Manuscrito aceptado: septiembre 15, 2020 